\title{
Dynamics and Fine Structures in Quiescent Prominences (MSDP/Pic du Midi, SOHO/SUMER and CDS)
}

Kejun $\mathrm{Li}^{2}$, J.-M. Malherbe ${ }^{1}$, J.E. Wiik ${ }^{4,5}$, B. Schmieder ${ }^{1,4}$, Th. Roudier ${ }^{3}$, T. Kucera ${ }^{6}$, and A. Poland ${ }^{6}$

Abstract. During the June 1996 campaign using the MSDP spectrograph at Pic du Midi and SUMER/CDS (JOP 12) on board SOHO, quiescent prominences were observed. We present observations and physical quantities of the June 5 prominence. Doppler shifts, temperatures and electron densities of fine structures were deduced from $\mathrm{H} \alpha$ data.

\section{Observations}

Observations of the prominence were performed on June 5,1996 between 09:30 to 11:00 UT in $\mathrm{H} \alpha$ with the MSDP spectrograph at Pic du Midi (11 simultaneous $2 \mathrm{D}$ channels of $4^{\prime} \times 30^{\prime \prime}$ ) together with the spectrographs on SUMER and CDS. Data were also recorded with the Meudon Spectroheliograph ( $\mathrm{H} \alpha$ and $\mathrm{CaII} \mathrm{K}$ ) and with the $\mathrm{H} \alpha$ coronograph of Wroclaw Observatory (Rompolt and Rudawy 1997, private comm., Wiik et al. 1997). Figure 1 shows a set of data obtained at Meudon (spatial resolution of 1.8") and Pic du Midi (0.5") for chromospheric temperatures, and by SUMER (1.5") for transition zone temperatures. We also have data from $C D S$ in various lines from $8 \times 10^{4} \mathrm{~K}$ to more than $10^{6} \mathrm{~K}$ (Figure 2).

We have to date worked essentially on $\mathrm{H} \alpha$ line profiles in order to derive physical parameters from spectroscopic diagnostics. The profiles in Figure 3 show symmetrical line profiles, which have been fitted by a least square method to a cloud model; asymmetrical line profiles have been fitted using a two-cloud model ( $\mathrm{Li}$ et al. 1994). We made the assumption that the source function can be considered as a constant if the optical thickness at the line center is less than unity. We derive with this method the line of sight velocity (Doppler shift), the column density of hydrogen at the second level, $N_{2}$ (from the Doppler width of the line and the optical thickness at line centre), the excitation temperature (from the source function and departure coefficients, see Gouttebroze et

\footnotetext{
${ }^{1}$ Observatoire de Paris, DASOP, 92195 Meudon, France

${ }^{2}$ Yunnan Observatory, Kunming, China

${ }^{3}$ Observatoire Midi Pyrénées, 65200 Bagnères de Bigorre, France

${ }^{4}$ University of Oslo, PO Box 1029, Blindern, 0315 Oslo, Norway

${ }^{5}$ Observatoire de Nice, BP 229, 06304 Nice Cedex 4, France

${ }^{6}$ NASA, GSFC, MD 20771, USA
} 

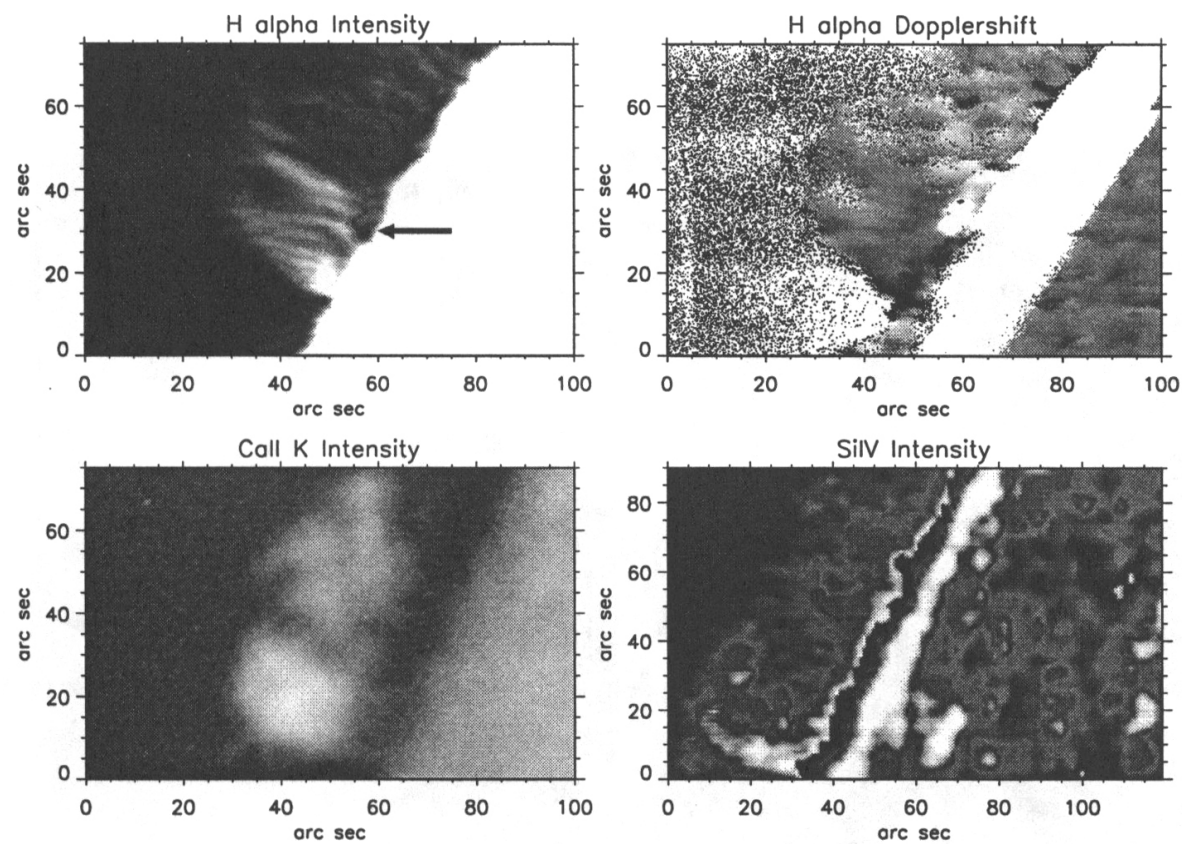

Figure 1. Observations. Top: MSDP/Pic du Midi; bottom left: Meudon; bottom right: SUMER/SOHO

al. 1993), and a microturbulent velocity, $V_{t}$ (assuming that the Doppler width is the combination of a thermal and non-thermal component). We also computed the electron density derived from the relationship between the emission measure, $\mathrm{E}$, and the integrated observed $\mathrm{H} \alpha$ intensity given by Heinzel et al. (1994). Values of $n_{e}$ were, hence, obtained by this method according to the equation, $n_{e}^{2} D=10^{28.9}$ (from observations), where $\mathrm{D}$ is the thickness of the structure. Results are presented in the tables for some pixel locations covering the prominence (in both tables, $N_{2}$ is expressed in terms of $10^{12} \mathrm{~cm}^{-2}$ ).

Table 1. Physical quantities of some symmetrical $\mathrm{H} \alpha$ line profiles

\begin{tabular}{lclccc}
\hline \hline Prof. & $V_{/ /}\left(\mathrm{kms}^{-1}\right)$ & $V_{t}\left(\mathrm{kms}^{-1}\right)$ & $T_{\text {ex }}(\mathrm{K})$ & $N_{2}$ & $E\left(\mathrm{ergs}^{-1} \mathrm{~cm}^{-2} \mathrm{sr}^{-1}\right)$ \\
\hline A & 3.93 & 15.9 & 7181 & 1.85 & $0.67 \times 10^{5}$ \\
B & 2.49 & 14.2 & 6742 & 2.35 & $0.63 \times 10^{5}$ \\
C & 3.65 & 14.6 & 9633 & 0.88 & $0.77 \times 10^{5}$ \\
D & 2.97 & 13.4 & 10405 & 0.62 & $0.66 \times 10^{5}$ \\
\hline
\end{tabular}

\section{Discussion and Conclusion}

Tables 1 and 2 show that reasonable values are obtained from the cloud model applied to symmetrical profiles. Table 3 shows the result of the two-cloud method 
applied to asymmetrical profiles and reveals the existence of two structures with opposite velocities.

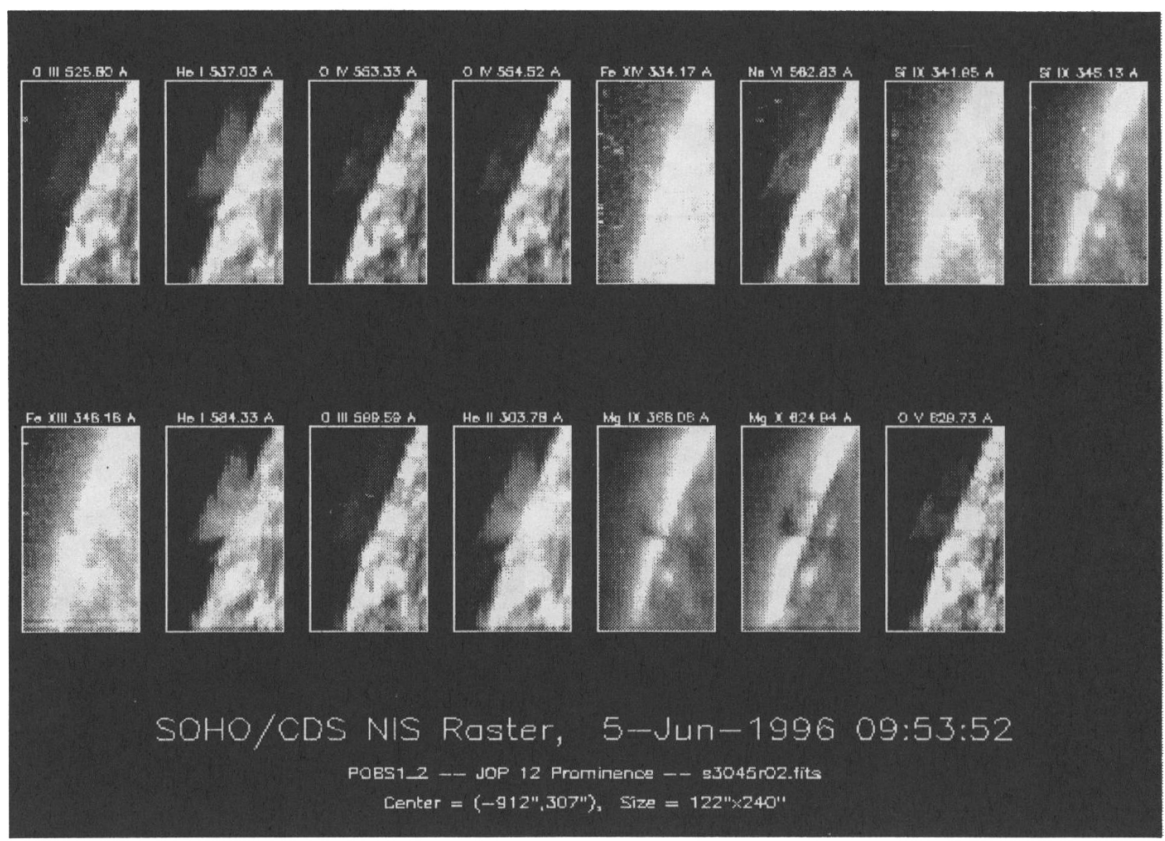

Figure 2. Observations in different lines with CDS/SOHO

Table 2. Electron density, $n_{e}$, variation with thread thickness, $D$, derived from NTLE relationship between emission measure, $\mathrm{E}$, and $\mathrm{H} \alpha$ intensity.

\begin{tabular}{llllllll}
\hline \hline$D(\mathrm{~km})$ & 1000 & 1500 & 2000 & 2500 & 3000 & 3500 & 4000 \\
$\log _{10} n_{2}$ & 4.15 & 3.98 & 3.85 & 3.75 & 3.68 & 3.61 & 3.55 \\
$n_{e}\left(\times 10^{10} \mathrm{~cm}^{-3}\right)$ & 2.82 & 2.30 & 1.99 & 1.78 & 1.63 & 1.51 & 1.41 \\
\hline
\end{tabular}

Table 3. Physical quantities of some asymmetrical lines

\begin{tabular}{lcccccccc}
\hline \hline Prof. & \multicolumn{3}{c}{ The first cloud } & \multicolumn{4}{c}{ The second cloud } \\
& $\begin{array}{c}V_{/ /} \\
V_{t}\end{array}$ & $T_{e x}$ & $N_{2}$ & $V_{/ /}$ & $V_{t}$ & $T_{\text {exc }}$ & $N_{2}$ \\
& $(\mathrm{~km} / \mathrm{s})$ & $(\mathrm{km} / \mathrm{s})$ & $(\mathrm{K})$ & & $(\mathrm{km} / \mathrm{s})$ & $(\mathrm{km} / \mathrm{s})$ & $(\mathrm{K})$ & \\
\hline 1 & -19.48 & 9.2 & 6970 & 0.33 & 2.38 & 13.7 & 7978 & 1.23 \\
2 & -9.75 & 8.0 & 17542 & 0.19 & 6.20 & 24.6 & 8272 & 6.74 \\
3 & -15.57 & 9.3 & 7080 & 0.20 & 2.49 & 14.5 & 7379 & 1.19 \\
4 & -11.39 & 10.6 & 16854 & 0.58 & 10.1 & 15.9 & 21563 & 0.90 \\
5 & -3.42 & 22.8 & 21853 & 1.15 & 15.8 & 9.7 & 9588 & 1.18 \\
6 & 9.29 & 21.2 & 15737 & 1.27 & -15.8 & 16.1 & 12871 & 1.45 \\
\hline
\end{tabular}

The different line profiles studied in Tables 1 and 3 are taken along a cross section of the prominence as indicated by an arrow in Figure 1. In particular, the asymmetrical profiles of Table 3 are observed in the " $\mathrm{H} \alpha$ black hole or cav- 

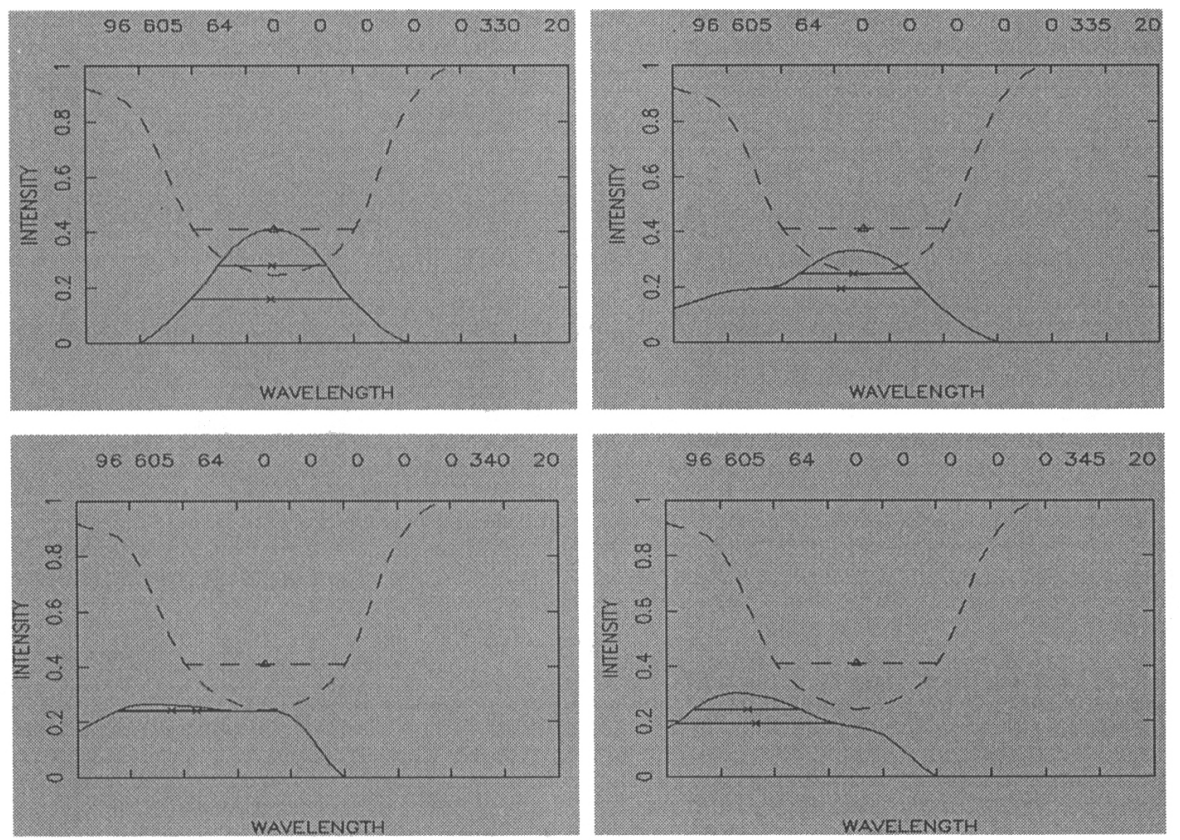

Figure 3. $\quad \mathrm{H} \alpha$ observed line profiles (MSDP/Pic du Midi; dashed line for mean chromospheric profile, continuous line for prominence profile). Both symmetric and asymmetric profiles are observed. The wavelength step between two consecutive channels is $0.26 \stackrel{\AA}{A}$.

ity" at the bottom of the prominence. This "cavity" was well observed between 09:50 and 10:05 UT. Opposite radial velocities (magnitudes of the order of 10 $\mathrm{km} / \mathrm{s}$ ) are found inside this structure using the two-cloud inversion, which seems to reveal the presence of two dynamically different threads. The temperatures derived from our calculations are typical for prominences $\left(10^{4} \mathrm{~K}\right)$ and the electron densities are of the order of $2 \times 10^{10} \mathrm{~cm}^{-3}$, in agreement with previous results (Leroy and Bommier 1997, private communication).

Acknowledgments. This work was performed while one of the authors (L. K.) was a visitor at Meudon Observatory. He is grateful to the Chinese Academy of Sciences for financial support.

\section{References}

Gouttebroze P., Heinzel P. and Vial J.-C. 1993, A\&AS, 99, 513

Heinzel P., Gouttebroze P. and Vial J.-C. 1994, A\&A, 292, 656

Li K. et al. 1994 Solar Phys., 150, 87

Wiik J.E. et al. 1997, JOSO Annual Report, 93 